\title{
Microfabrication by optical tweezers
}

R. Ghadiri, T. Weigel, C. Esen, A. Ostendorf

R. Ghadiri, T. Weigel, C. Esen, A. Ostendorf, "Microfabrication by optical tweezers," Proc. SPIE 7921, Laser-based Micro- and Nanopackaging and Assembly V, 792102 (21 February 2011); doi: 10.1117/12.887264 


\title{
Microfabrication by Optical Tweezers
}

\author{
R. Ghadiri, T. Weigel, C. Esen, A. Ostendorf* \\ Ruhr-University Bochum, Institute of Laser Applications Technology, Universitätsstr. 150, 44801 \\ Bochum, Germany
}

\begin{abstract}
A new method to fabricate microstructures built by polymer microparticles using a bottom-up technique is presented. The microstructures find broad application in micro-fluidics technology, photonics and tissue-engineering. The handling of the particles is realized by a holographic optical tweezers setup, ensuring the precise allocation of the particles to the desired structure. A biochemical technique ensures that the structure remains stable independent of the laser source. We show that with this method complex two-dimensional durable structures can be assembled and cannot be separated by optical forces. The structures are extendable during the entire fabrication process and can be linked to further particles and structures as desired.
\end{abstract}

Keywords: optical tweezers, optical trapping, holographic optical tweezers, micro assembling, micromanipulation, microfabrication, micromachining

\section{INTRODUCTION}

Microstructures find their utilization in a wide field of engineering and medical technology. In microfluidic applications [1] small structures are utilized such as micro valves to control fluid and particle flow. Especially tailored tools can be helpful for the manipulation of small objects within defined chambers. In photonics engineering microparticles arranged to periodic structures are utilized to guide light within very small scales (photonic crystals) [2]. Due to the different refractive index between particles and the surrounding medium the change of refractive index along the structure can be manipulated by the appropriate constellation of the particles, being the crucial point to be considered for the light propagating characteristics. The third main domain where microstructures are essential is the field of tissue engineering $[3,4]$. Special scaffolds are needed to locate living cells and to enable cell growth. A technique ensuring the manufacturing of structures simultaneous to the cell manipulation process would be convenient.

One promising approach in manufacturing microstructures is assembling micro beads to the desired structure assisted by optical tweezers (OT) $[5,6]$. This approach contains two tasks: First we need a technique to trap and move the microparticles to allocate them in the desired constellation. For the manipulation of particles optical tweezers seem to be a particularly suitable tool, facilitating the precise arrangement of small objects to complex structures [7]. This technique is also suitable in combination with biological objects, as it benefits from the contact free and harmless character. For an efficient particle assembling technique, the control of several particles at the same time is necessary. Nowadays mainly two classes of optical trapping techniques compete in multiple particle manipulation: Scanning optical tweezers (SOT) $[8,9]$ use a time-shifting principle to switch between the positions of each particle to be trapped. High speed components like tilt mirrors (TM) or acousto-optic deflectors (AOD) enable the simultaneous trapping and manipulation of several particles. Nevertheless SOTs are usually limited to manipulating particles in the plane perpendicular to the laser beam propagation, i.e. in two dimensions. In contrast holographic optical tweezers (HOT) $[10,11]$ permit to trap and shift objects three dimensionally independent for each particle. For this purpose the laser beam is modulated by spatial light modulators (SLM) in an appropriate way. Furthermore this technique allows for manipulating objects with different optical properties, e.g. reflecting and absorbing particles.

\footnotetext{
* andreas.ostendorf@ruhr-uni-bochum.de; Phone: +49-234-32-25233; Fax: +49-234-32-14529; www.lat.rub.de
} 
The second task for the micro manufacturing process is to provide a binding technique, which is appropriate for the used material and provides durable structures independent of the laser beam. Several approaches have been presented as applying thermal processes to bind polymer particles together or using the photopolymerization mechanism to enable particle binding. In our experiments we use biomolecules with high affinities as particle coatings to ensure the durable assembling of particles. We show how complex structures can be produced in a simple way with the option of expanding them by further particles or linking with other sub-structures. This method is also suitable for three dimensional structures.

\section{PARTICLE MANIPULATION}

\subsection{Principle of optical tweezers}

Optical tweezers use the optical forces arising, when a tightly focused laser beam interacts with a particle. Using a high numerical aperture (NA) objective the emerging gradient forces dominate the process. Assuming particle diameters much larger than the used laser wavelength, the effect can be explained by geometrical optics. While a transparent bead is illuminated by a focused laser beam, the light is reflected and refracted at the surface of that bead due to the different refractive index causing a change of propagation direction. Considering that light can be described as a photon flux with each photon carrying a momentum, this change of momentum for any ray has to be compensated by another momentum transferred to the particle due to momentum conservation. This transferred momentum is directed contrarily to the momentum change $\Delta \mathbf{p}=\mathbf{p}_{\text {out }}-\mathbf{p}_{\text {in }}$, determined by the incident and emergent light (figure 1 a) and corresponds to a force acting on the sphere. In the most common case of particles exhibiting a higher refractive index than the surrounding medium ( $\mathrm{n}_{\text {particle }}>\mathrm{n}_{\text {medium }}$ ), summing up all arising forces from each ray will result in a net force accelerating the particle to the maximum of the beam intensity. If the particle is laterally displaced from the beam center, the net force pulls the bead into the center of the beam (figure 1 a). If the particle is displaced axially, the net forces will accelerate the bead to a stable position near the beam waist (figure $1 \mathrm{~b}$ ). In this position the net force acting on the particle equals zero. Depending on parameters like the applied laser power, the relative refractive index $n=n_{\text {particle }} / n_{\text {medium }}$ and the numerical aperture of the microscope objective, these forces acting on a microbead typically lie in the range of 1 to $100 \mathrm{pN} \mathrm{[12].} \mathrm{To}$ actively move particles an appropriate beam deflection technique is necessary.

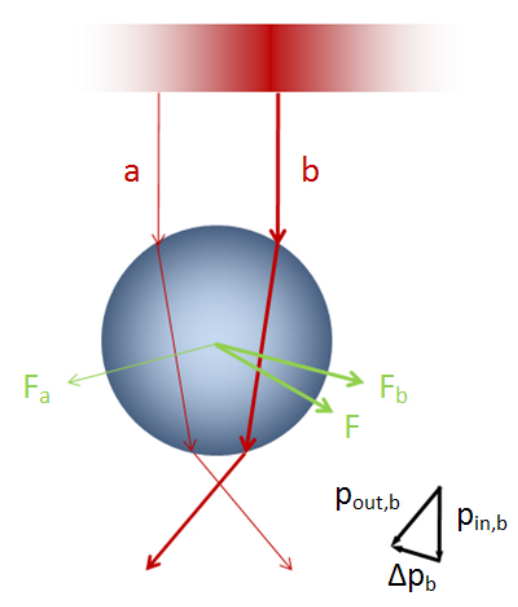

a)

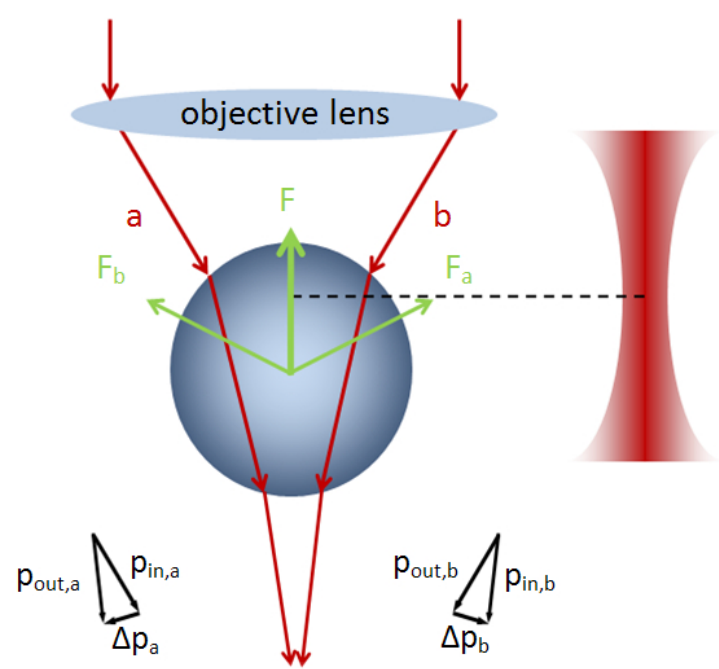

b)

Figure 1. Optical Forces arising due to refraction at the particle surface and momentum transfer. a) Particle laterally accelerated to the point of highest beam intensity. b) Particle forced to the stable position near the beam waist. 
By slightly deflecting the laser spot position, the particle will follow the laser beam, until it reaches the stable position and can be moved from one position to another one. In the most intuitive way this is achieved by manual or motorized tilt mirrors. Alternatively also the particles containing chamber, usually fixed to a motion stage, can be moved relative to the laser beam position. To trap and manipulate several particles simultaneously scanning methods (SOT - scanning optical tweezers) can be used to switch the laser beam between several positions of the particles rapidly. Hereby a single laser beam is time-shared and the entire intensity is divided to the different positions of the particles to be trapped. Is the scanning rate faster than the Brownian relaxation time several particles can be stably trapped against thermal motion [12]. This high speed beam deflection is usually realized by motorized tilt mirrors or by acousto-optic modulators (AOM). Multiparticle trapping was realized successfully and up to 400 traps have been reported by Vossen et. al. [13]. Still SOTs suffer from drawbacks as they are generally limited to planar trap constellations and to transparent objects. Also the instantaneous beam power necessary to trap particles stably is considerably high while trapping multiple objects, as the trap strength depends on the mean intensity affecting one particle.

\subsection{Holographic optical tweezers}

A more convenient and versatile way to manipulate multiple particles is the technique of holographic optical tweezers (HOT). Spatial light modulators (SLM) are used to shape the incident laser light and to split it into multiple beams each one able to trap one particle. As the trap strength depends on the average power, less peak intensities are required to ensure the same forces as in the case of scanning techniques. This leads to less damaging, particularly important while working with biological probes. It has been shown that up to more than hundred optical traps can be generated by using this technique [7, 10]. Furthermore HOTs are not limited to planar trap configurations. Several particles can be trapped in different planes independently just by applying the appropriate beam shaping with the SLM [7]. Converting an ordinary Gaussian beam into a Laguerre-Gaussian mode enables to also manipulate opaque objects with so-called optical vortices [14]. Among numerous types of SLMs phase modulating models have become quite popular because of their relatively high efficiency compared to amplitude modulating versions. They mostly consist of a liquid crystal display (LCD), whose each pixel is able to retard the incident wave front depending on the applied voltage at this pixel. This is done by the influence of the electrical field arranging the liquid crystal molecules impacting the optical density of the medium. By this means the optical path of the light is manipulated and the incident wave front locally retarded. The holograms projected on the SLM are computer generated by iterative algorithms, e.g. the Gerchberg-Saxton algorithm [15] or the Direct Search algorithm [16]. Each algorithm benefits from advantages regarding the quality of the generated beam intensity or the calculation efficiency. An extensive comparison between the performance of different algorithms can be found in [17]. Every calculated phase hologram -also called kinoform- encodes the information about one 3D trap arrangement in a grayscale image, each gray level converted to a voltage and thus manipulating the local phase retardation. By replacing a hologram by another one representing slightly displaced traps the particles will be moved into the new configuration. The SLM can update the imposed holograms at video rates enabling the independent moving of each trap through the medium. In this manner particles can be controlled and assembled to the desired structure for the duration of the active laser beam (figure 2).

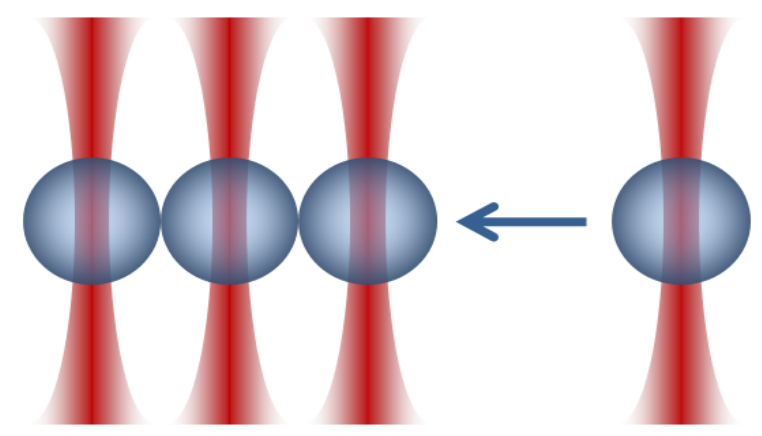

Figure 2. Micromanipulation with holographic optical tweezers. Particles can be arranged to a temporary structure.

Once the laser is switched off, the particles will lose the imposed alignment. 
As soon as the laser source is switched off the particles will lose the imposed arrangement and follow statistical movements provoked by Brownian motion. To ensure permanent structures an appropriate binding technique has to be applied as will be discussed in chapter 3. For more comfortable handling of particles we use the software BlueTweezers from the Optics research group of the University of Glasgow [18] with the option to choose from the common algorithms. This program delivers a user-friendly interface to interactively trap and move particles. Several spots can be generated simultaneously and moved in an appropriate way to assemble particles via drag and drop.

\subsection{Experimental setup}

For the manipulation of multiple particles we use a HOT setup as illustrated in figure 1. In our setup we apply an infrared laser source with an emitting wavelength of $\lambda=1030 \mathrm{~nm}$ and a beam diameter of $2 \mathrm{~mm}$ (ELS - VersaDisk). The maximum output power is $10 \mathrm{~W}$. For the beam shaping we use a phase modulating reflective SLM (Holoeye Pluto NIR). A beam expansion is necessary to not damage the SLM and also to allow using the entire active area of the device. The beam is expanded by a 10x beam expander to considerably overfill the SLM. The lenses L1 and L2 form a second telescope ( $\mathrm{f} 1=300 \mathrm{~mm}, \mathrm{f} 2=160 \mathrm{~mm}$ ), for adjusting the diameter of the reflected beam to the aperture of the microscope objective (Carl Zeiss, Plan-Apochromat, 100x/1.40 Oil DIC) and to project the hologram onto the back focal plane of the microscope objective. The beam is coupled into an inverted microscope (Axiovert $135 \mathrm{TV}$, Carl Zeiss) and guided into the objective by a dichroic mirror, which reflects the infrared light and transmits the visible spectrum. The desired beam intensity pattern is first generated in the focal plane of the lens L1. If necessary, a filtering of the beam intensity can be applied at this plane, e.g. to filter out the zeroth diffraction order, caused by the non-perfect character of the pixelated SLM display structure. Following the final intensity pattern is generated in the focal plane of the microscope objective, where the particles are located in an appropriate chamber. The chamber is built by an aluminum part of $10 \mathrm{~mm}$ thickness containing a circular aperture with the diameter of $10 \mathrm{~mm}$. The aperture is closed with two cover glasses on the top and the bottom, each sealed with silicone gel. The chamber is mounted to a motorized positioning stage (Luigs \& Neumann) for moving the probe over long distances.

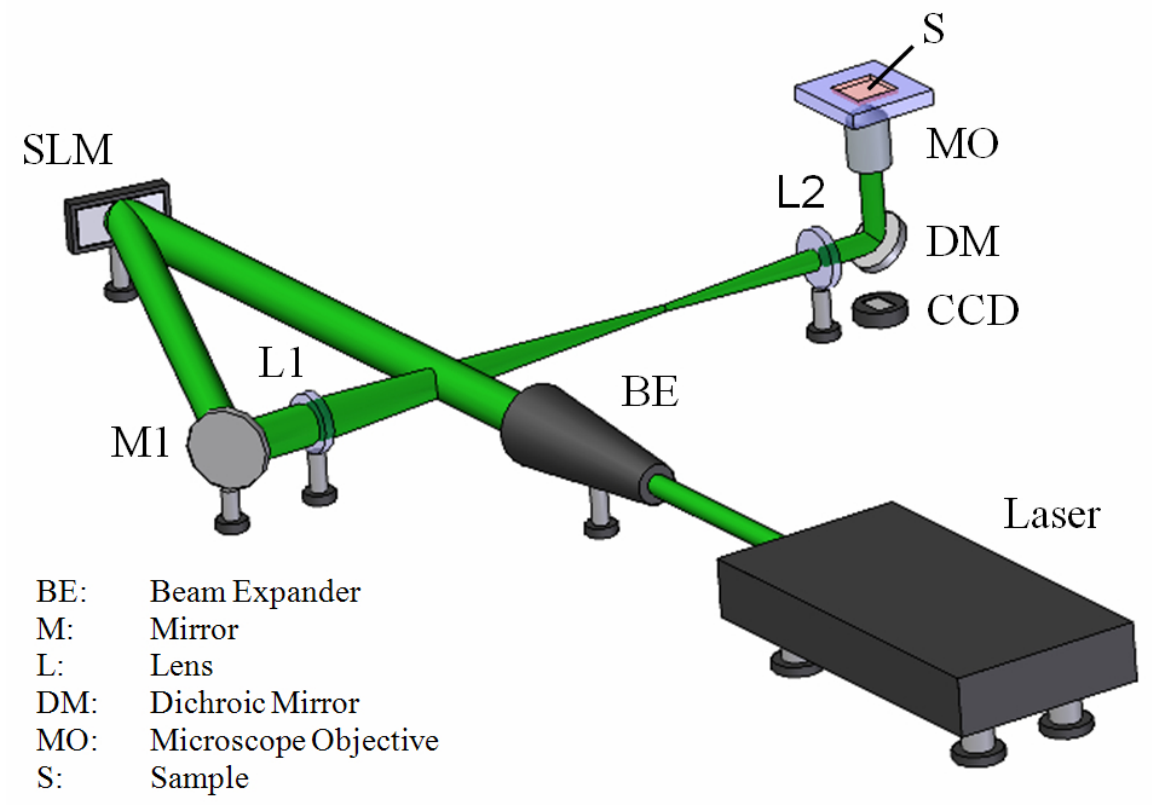

Figure 3. Experimental setup including a Yb:YAG laser (ELS - Versadisk, $\lambda=1030 \mathrm{~nm}$ ), a spatial light modulator

(Holoeye Pluto NIR) and a high numerical aperture microscope objective (Zeiss Plan-Apochromat, NA = 1.4). 
More details about optimization the HOT setup have extensively been discussed by Martin-Badosa [19]. The particle manipulation and manufacturing process can be observed in-situ with an integrated CCD-Camera (EHDkam Pro2IR) through the same objective as used for trapping the particles.

\section{BINDING TECHNIQUE}

Several approaches have been proposed for the permanent binding of microparticles. Polymer particles can be linked via thermal and photothermal processes by melting the outer layer of the particles. The heat can be induced by laser irradiation absorbed on Rhodamine-B dyed polymer films [20] or aided by an external heating source [21]. Also photochemical reactions can be used to fix particles dispersed in a polymerizable solution together [22, 23]. In this approach one laser operating in the infrared was used to trap the microbeads while another pulsed UV-laser linked the particles together. Another approach has been presented by Terray, who utilized the same laser for trapping and connecting the particles [24]. By this means linear structures were manufactured and utilized as valves in microfluidics applications. Nevertheless all these approaches suffer from different constraints. Working in heated environments can lead to undesired conditions such as convection currents, generally complicating the assembling process. Furthermore the material can be damaged by heating effects, especially if the technique is applied combined with biological probes, e.g. living cells. Also the chemicals used in the photopolymerization approach are usually not biocompatible.

In our approach we use the strong affinity between the protein streptavidin (SA) and the corresponding vitamin biotin (B). If different particles are coated with these biomolecules they will bind with each other once they are joined together. The strong binding between these molecules is caused by the complementary structure of the binding pocket of SA and B (figure 4). Furthermore hydrogen bonds are formed during the reaction contributing to the binding strength.

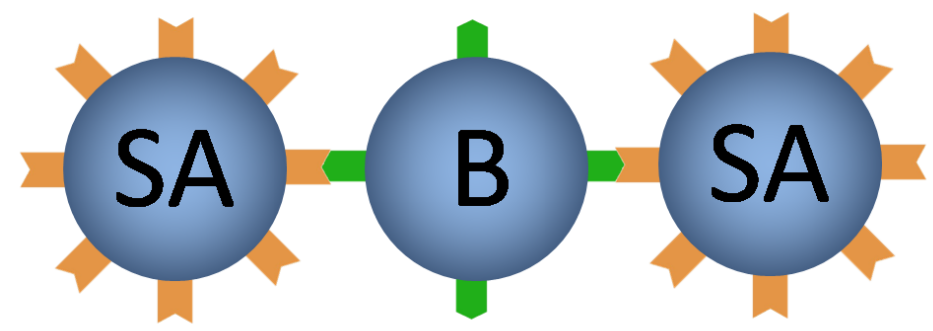

Figure 4. Simplified model for microparticle assembling using biomolecules. Complementary structures next to hydrogen bonds lead to the strong binding forces between Streptavidin and Biotin.

In fact the streptavidin-biotin binding is the strongest non-covalent binding known. Dissociation constants of $\mathrm{D}_{\mathrm{s}}=4 \times 10^{-14} \mathrm{M}$ have been reported by Holmberg [25]. Ota et. al. measured the rupture force of SA-B bonds using optical tweezers while pulling B coated particles from a SA coated substrate [26]. They determined forces in the range of 3.6 to $5.4 \mathrm{pN}$ with a loading rate of $7.7 \mathrm{pN} / \mathrm{s}$. Further discussions about the bonding forces and experimental determining of the rupture forces in SA-B binding can be found in [27, 28]. The binding between SA and B occurs very rapidly and is stable also under large variations in $\mathrm{pH}$ and temperature. The SA-B binding is widely used in biotechnology for biosensor applications or molecule separation [29]. One way to use these molecules to build microstructures was proposed by Castelino in 2005 [30] and implemented by Park two years later [31]. For this purpose B coated particles were optically trapped and arranged in the desired constellation. Subsequently SA molecules were added to the chamber to start the binding process. The SA molecules are delivered by the convection within the probe due to the heat induced by the laser beam and bind to the surface of the B coated particles. After some seconds the particles were linked together and could not be separated anymore. In this way two-dimensional structures like pyramids or micro tools could be manufactured. Adding more particles to the fabricated structure is not possible as long as the SA molecules are still situated in the processing chamber. To perform the assembling in a more flexible way, we use SA coated particles in combination with B coated microparticles to actively bind the building blocks together. As the coating with nanometer-sized molecules is 
not visible under the optical microscope, we rather use SA coated and B coated particles exhibiting considerably different sizes to manufacture microstructures (figure 5). By this means the linking parts are transported actively to the desired position of the particles to be connected and glued together. The reaction takes place in a more controlled way and SA-B binding becomes observable. After one structure has been manufactured, further fabricated sub-structures can be connected to each other to form more complex structures. In combination with HOTs this method could also be used for manufacturing sophisticated three dimensional structures.

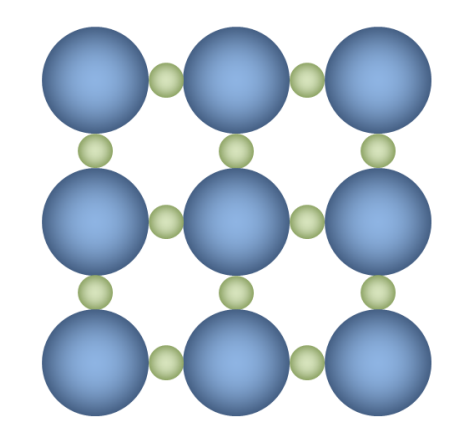

Figure 5. Model for manufacturing microstructures. Small Biotin coated particles are used as binding parts to connect the larger Streptavidin coated particles.

\section{EXPERIMENT}

In our experiments we choose SA coated polystyrene particles with a mean diameter $\emptyset_{\mathrm{SA}}=12 \mu \mathrm{m}$ and $\mathrm{B}$ coated particles with $\emptyset_{\mathrm{B}}=3.5 \mu \mathrm{m}$ (Kisker Biotech) as binding elements. The differently coated particles can be distinguished clearly under the optic microscope. To reduce the likelihood of uncontrolled self-assembly it is important to choose a sufficiently low particle concentration. For this we dilute $50 \mu \mathrm{l}(0.5 \% \mathrm{w} / \mathrm{v})$ of the SA particles in $1 \mathrm{ml}_{2} \mathrm{O}$ and $30 \mu \mathrm{l}(0.5 \% \mathrm{w} / \mathrm{v})$ of the B particles in $1 \mathrm{ml} \mathrm{H}_{2} \mathrm{O}$ separately. In all experiments particles were washed by a centrifugation method [32] to clean the particles from additives. After washing we add an amount of $0.1 \%$ of surfactant (Tween20) to the probe to reduce non-specific binding-effects between the particles due to hydrophobic interactions and particle sticking on the cover glass. Furthermore we sonicate all probes for two minutes before using them to reverse potential particle agglomeration. For each experiment we introduce $50 \mu \mathrm{l}$ of the diluted SA probe into the chamber and wait for a while, so that the SA particles can sediment to the bottom of the chamber, e.g. on the bottom cover glass. Then we add another $50 \mu \mathrm{l}$ of the B probe into the chamber. We can observe under the microscope how the smaller particles sediment and their concentration in the lower level of the chamber increases gradually. As soon as enough binding parts are available the manufacturing process can be initialized. While the number of particles sticking to the cover glass is reduced by the use of surfactant, still a notable number of sticking particles can be observed which are useless for the manufacturing process. Suitable particles in the vicinity of the assembled structure are trapped using the HOT, while those outside the field of view (approximately $45 \times 65 \mu \mathrm{m}$ ) have to be searched and approached with the aid of the motion stage.

\section{RESULTS}

To exclude that the assembling is caused by non-specific binding, we first trap two SA particles and join them together. Separating the SA-SA assemble is performed successfully even with the lowest laser power applied. The same way B particles behave, when they are approximated to each other. In contrast, once a SA-B couple is joined together, it cannot be separated anymore by the applied optical forces. To build the desired structure modeled in figure 5 we start trapping an arbitrary SA particle and locate it to the desired position. While one SA particle is hold, another B particle is approximated with a second trap and attached to the target particle (figure 6 a). We observe that the binding reaction 
takes place very fast, as the B particle cannot be separated, even after a fraction of a second. The conjunction can be rotated and displaced elsewhere within the chamber. Then we drag the next SA particle and link it to the assembled pair of particles (figure $6 \mathrm{~b}$-c). After building the vertical linear structure (figure $6 \mathrm{~d}$-e) further linking parts are added to one side (figure $6 \mathrm{f}$ ), so that the next SA particles can be attached. The vertical structure is rotated by $90^{\circ}$ and translated for allocation the structure in an appropriate position away from sticking particles. Following the second and the third lines are assembled (figure $6 \mathrm{~g}$-i) successively as described. The completed structure (figure 6 i) can still be moved and rotated entirely, e.g. by gripping two outer particles by two optical traps and repositioning them. Throughout the manufacturing procedure we tried to separate the structure during different process steps. Once the first two SA beads were connected by one B coated particle we tried to separate the structure by pulling the SA particles with two generated optical traps. Also having assembled three SA particles we attempted to separate the structure the same way pulling the outer beads. In both cases we observed that the structures resisted the applied optical forces and could not be separated.

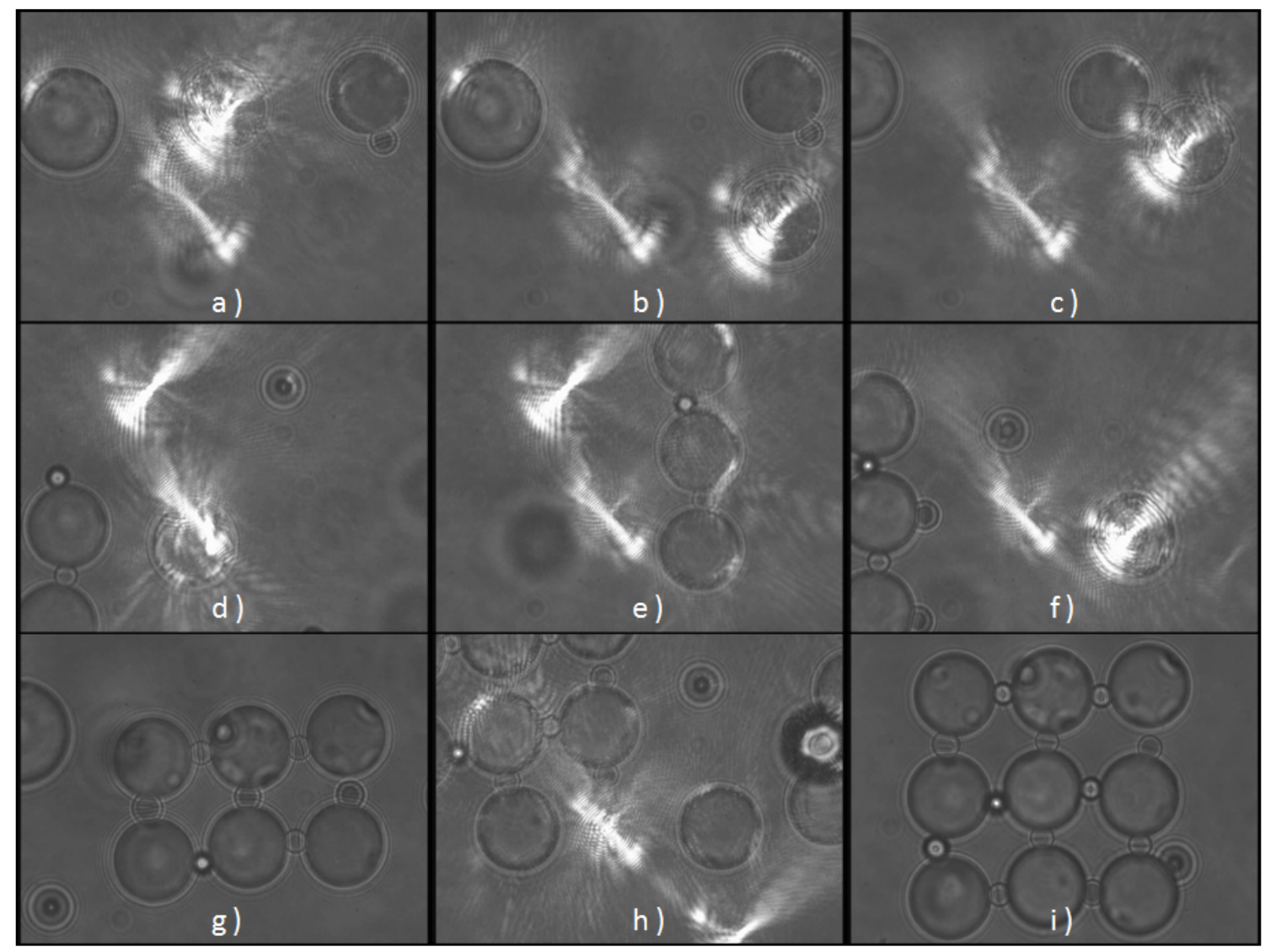

Figure 6. Micro manufacturing of a $3 \times 3$ array using Streptavidin coated polystyrene particles $\left(\varnothing_{\mathrm{SA}}=12 \mu \mathrm{m}\right)$ and Biotin coated particles $\left(\emptyset_{\mathrm{B}}=3.5 \mu \mathrm{m}\right)$ as binding parts. The laser beam position is visible due to reflection of the light at the glass water interface.

\section{CONCLUSION}

In this work we presented a method for the manufacturing of polymer microstructures based on polystyrene microparticles. The beads are used as building blocks, manipulated and arranged with the help of holographic optical tweezers. For the permanent binding of the desired structure we used biomolecules with a high affinity to each other. Streptavidin and biotin have been used as coatings on different sized particles. Hereby differently coated particles can be distinguished clearly during the binding process. Once the particles are joined the reaction occurs immediately and the 
particles are glued together. By this means a flexible way of microstructure manufacturing is given, as the built structures can be extended by more particles or further fabricated forms. Also the constructions are mobile during the manufacturing process and can be translated and rotated as desired. This method also can be used for future work on manufacturing three dimensional structures.

\section{ACKNOWLEDGEMENT}

We would like to thank the rector's office of the Ruhr-Universität Bochum for their generous support within the "Funds for high-risk scientific projects".

\section{REFERENCES}

[1] Whitesides, G. M., "The origins and the future of microfluidics," Nature 442(7101), 368-373 (2006).

[2] Joannopoulos, J. D., Villeneuve, P. R., and Fan, S. H., "Photonic crystals: Putting a new twist on light," Nature 386(6621), 143-149 (1997).

[3] Hutmacher, D. W., "Scaffolds in tissue engineering bone and cartilage," Biomaterials 21(24), 2529-2543 (2000).

[4] Yang, S. F., Leong, K. F., Du, Z. H., and Chua, C. K., "The design of scaffolds for use in tissue engineering. part ii. rapid prototyping techniques," Tissue Engineering 8(1), 1-11 (2002).

[5] Ashkin, A., "Acceleration and trapping of particles by radiation pressure," Physical Review Letters 24(4), 156-159 (1970).

[6] Ashkin, A., Dziedzic, J. M., Bjorkholm, J. E., and Chu, S., "Observation of a single-beam gradient force optical trap for dielectric particles," Optics Letters 11(5), 288-290 (1986).

[7] Curtis, J. E., Koss, B. A., and Grier, D. G., "Dynamic holographic optical tweezers," Optics Communications 207(1-6), 169-175 (2002).

[8] Mio, C., Gong, T., Terray, A., and Marr, D. W. M., "Design of a scanning laser optical trap for multiparticle manipulation," Review of Scientific Instruments 71(5), 2196-2200 (2000).

[9] Visscher, K., Brakenhoff, G. J., and Krol, J. J., "Micromanipulation by multiple optical traps created by a single fast scanning trap integrated with the bilateral confocal scanning laser microscope," Cytometry 14(2), 105-114 (1993).

[10] Grier, D. G. and Roichman, Y., "Holographic optical trapping," Applied Optics 45(5), 880-887 (2006).

[11] Burnham, D. R. and McGloin, D., "Holographic optical trapping of aerosol droplets," Optics Express 14(9), 41754181 (2006).

[12] Neuman, K. C. and Block, S. M., "Optical trapping," Review of Scientific Instruments 75(9), 2787-2809 (2004).

[13] Vossen, D. L. J., van der Horst, A., Dogterom, M., and van Blaaderen, A., "Optical tweezers and confocal microscopy for simultaneous three-dimensional manipulation and imaging in concentrated colloidal dispersions," Review of Scientific Instruments 75(9), 2960-2970 (2004).

[14] He, H., Friese, M. E. J., Heckenberg, N. R., and Rubinsztein-Dunlop, H., "Direct observation of transfer of angularmomentum to absorptive particles from a laser-beam with a phase singularity," Physical Review Letters 75(5), 826829 (1995).

[15] Gerchberg, R. and Saxton, W. O., "Practical algorithm for determination of phase from image and diffraction plane pictures," Optik 35(2), 237-246 (1972).

[16] Meister, M. and Winfield, R. J., "Novel approaches to direct search algorithms for the design of diffractive optical elements," Optics Communications 203(1-2), 39-49 (2002).

[17] Di Leonardo, R., Ianni, F., and Ruocco, G., "Computer generation of optimal holograms for optical trap arrays," Optics Express 15(4), 1913-1922 (2006).

[18] http://www.physics.gla.ac.uk/Optics/projects/tweezers/software/.

[19] Martin-Badosa, E., Montes-Usategui, M., Carnicer, A., Andilla, J., Pleguezuelos, E., and Juvells, I., "Design strategies for optimizing holographic optical tweezers set-ups," Journal of Optics a-Pure and Applied Optics 9(8), S267-S277 (2007).

[20] Won, J., Inaba, T., Masuhara, H., Fujiwara, H., Sasaki, K., Miyawaki, S., and Sato, S., "Photothermal fixation of laser-trapped polymer microparticles on polymer substrates," Applied Physics Letters 75(11), 1506-1508 (1999). 
[21] Ghadiri, R., Surbek, M., Esen, C., and Ostendorf, A., "Optically based manufacturing with polymer particles," Physics Procedia 5, 47-51 (2010).

[22] Misawa, H., Sasaki, K., Koshioka, M., Kitamura, N., and Masuhara, H., "Multibeam laser manipulation and fixation of microparticles," Applied Physics Letters 60(3), 310-312 (1992).

[23] Misawa, H., Sasaki, K., Koshioka, M., Kitamura, N., and Masuhara, H., "Laser manipulation and assembling of polymer latex-particles in solution," Macromolecules 26(2), 282-286 (1993).

[24] Terray, A., Oakey, J., and Marr, D. W. M., "Fabrication of linear colloidal structures for microfluidic applications," Applied Physics Letters 81(9), 1555-1557 (2002).

[25] Holmberg, A., Blomstergren, A., Nord, O., Lukacs, M., Lundeberg, J., and Uhlen, M., "The biotin-streptavidin interaction can be reversibly broken using water at elevated temperatures," Electrophoresis 26(3), 501-510 (2005).

[26] Ota, T., Sugiura, T., and Kawata, S., "Rupture force measurement of biotin-streptavidin bonds using optical trapping," Applied Physics Letters 87(4), 043901 (2005).

[27] Grubmuller, H., Heymann, B., and Tavan, P., "Ligand binding: Molecular mechanics calculation of the streptavidin biotin rupture force," Science 271(5251), 997-999 (1996).

[28] Moy, V. T., Florin, E. L., and Gaub, H. E., "Intermolecular forces and energies between ligands and receptors," Science 266(5183), 257-259 (1994).

[29] Girault, S., Chassaing, G., Blais, J. C., Brunot, A., and Bolbach, G., "Coupling of maldi-tof mass analysis to the separation of biotinylated peptides by magnetic streptavidin beads," Analytical Chemistry 68(13), 2122-2126 (1996).

[30] Castelino, K., Satyanarayana, S., and Sitti, M., "Manufacturing of two and three-dimensional micro/nanostructures by integrating optical tweezers with chemical assembly," Robotica 23, 435-439 (2005).

[31] Park, I. Y., Sung, S. Y., Lee, J. H., and Lee, Y. G., "Manufacturing micro-scale structures by an optical tweezers system controlled by five finger tips," Journal of Micromechanics and Microengineering 17(10), N82-N89 (2007).

[32] http://www.bangslabs.com/literature/technotes. 